\title{
Os Répteis do Município de São Paulo: diversidade e ecologia da fauna pretérita e atual
}

\author{
Otavio Augusto Vuolo Marques ${ }^{1}$, Donizete Neves Pereira ${ }^{1,3}$, Fausto Erritto Barbo ${ }^{1,4}$, \\ Valdir José Germano² \& Ricardo Jannini Sawaya ${ }^{1}$ \\ ${ }^{1}$ Laboratório Especial de Ecologia e Evolução, Instituto Butantan, \\ Av. Dr. Vital Brasil, 1500, CEP 05503-900, São Paulo, SP, Brasil \\ ${ }^{2}$ Laboratório de Herpetologia, Instituto Butantan, \\ Av. Dr. Vital Brasil, 1500, CEP 05503-900, São Paulo, SP, Brasil \\ ${ }^{3}$ Programa de Pós Graduação Interunidades em Biotecnologia, Instituto de Ciências Biomédicas, \\ Universidade de São Paulo - USP. \\ Av. Prof. Lineu Prestes, 1730. Ed. ICB-IV, Ala Norte, sala 3, \\ Cidade Universitária, CEP 05508-900, São Paulo, SP, Brasil \\ ${ }^{4}$ Programa de Pós Graduação em Biologia Animal, Instituto de Biociências, Letras e Ciências Exatas, \\ Universidade Estadual Paulista - UNESP, \\ Rua Cristóvão Colombo, 2265, CEP 15054-000, CEP 15054-000, São José do Rio Preto, SP, Brasil \\ ${ }_{5}^{5}$ Autor para correspondência: Otavio Augusto Vuolo Marques, e-mail: otaviomarques@butantan.gov.br
}

MARQUES, O.A.V., PEREIRA, D.N., BARBO F.E., GERMANO, V.J. \& SAWAYA, R.J. Reptiles in São Paulo municipality: diversity and ecology of the past and present fauna. Biota Neotrop., $9(2)$ : http://www. biotaneotropica.org.br/v9n2/en/abstract?article+bn02309022009.

\begin{abstract}
The reptile fauna in the municipality of São Paulo is well sampled due to intensive collection in the last 100 years. In the present work we provide a checklist of reptile species in São Paulo municipality based on preserved specimens in scientific collections. The reptile fauna was also characterized by three ecological parameters: habitat use, substrate use, and diet. We recorded a total of 97 reptile species (two turtles, one crocodilian, 19 lizards, seven amphisbaenians, and 68 snakes). Approximately $70 \%$ of the lizards and $40 \%$ of the snakes are typical of forest habitats of the Serra do Mar mountain range. Other squamates are typical of open formations that occur mainly on inland Cerrado habitats. All turtles and the crocodilian are associated to riparian habitats. Approximately $63 \%$ of the lizards are predominantly terrestrial, and the remaining species are arboreal. Most species of snakes are terrestrial (38\%) or subterranean/criptozoic $(25 \%)$ whereas a smaller proportion are arboreal $(18 \%)$ or aquatic (9\%). Lizards feed upon arthropods. Almost 50\% of the snake species are specialized or feed mainly upon anuran amphibians. Other important items consumed by snakes are mammals $(24 \%)$, lizards (18\%), subterranean vertebrates (10\%), and invertebrates (earthworms, mollusks and arthropods; $15 \%$ ). A total of 51 reptile species have not been recorded for the last six years. Probably many of these species are extinct in the region due the intense local urbanization and habitat loss. The survey of species collected in São Paulo municipality and received in the Instituto Butantan in recent years allowed the identification of 10 lizards at least 42 snake species already occurring in the region. The high species richness of the original fauna seems related to the geographic location of the municipality, in a contact zone between forested areas of the Atlantic Forest (ombrophilous forest) and open formations (savannas, high-altitude grassland). Thus, the original habitat composition probably allowed sympatry among different species pools typical of both open and forested formations. The extant snake fauna recorded in the last three years indicates a higher loss of the species in open formations when compared to the forested areas.
\end{abstract}

Keywords: reptiles, diversity, municipality of São Paulo, richness, ecological tendency.

MARQUES, O.A.V., PEREIRA, D.N., BARBO F.E., GERMANO, V.J. \& SAWAYA, R.J. Os Répteis do Município de São Paulo: diversidade e ecologia da fauna pretérita e atual. Biota Neotrop., 9(2): http://www. biotaneotropica.org.br/v9n2/pt/abstract?article+bn02309022009.

Resumo: O Município de São Paulo é uma área bem amostrada em relação à fauna de répteis devido à coleta intensiva feita pela população local nos últimos 100 anos. Neste trabalho consultamos registros e examinamos exemplares de coleções científicas para elaborar uma lista das espécies da região. A fauna de répteis também foi caracterizada em relação a três parâmetros ecológicos: uso do ambiente, uso de substrato e hábitos alimentares. Registramos um total de 97 espécies de répteis (dois quelônios, um crocodiliano, 19 lagartos, sete anfisbenídeos e 68 serpentes). Aproximadamente $70 \%$ da fauna de lagartos e $40 \%$ das serpentes é composta por espécies típicas de ambientes florestais e ocorrem na Serra do Mar. Outros squamata são característicos de formações abertas e são encontrados, sobretudo nas formações abertas de Cerrado do interior paulista. Todas tartarugas e o crodiliano 
estão associados a corpos da água. Aproximadamente 63\% dos lagartos são predominantemente terrícolas e os demais são arborícolas. A maior parte das espécies de serpentes é terrícola (38\%) ou subterrânea/criptozóica (25\%), menor parte é arborícola (18\%) ou aquática (9\%). Artrópodes são o item predominante na dieta de lagartos. Quase 50\% das espécies de serpentes alimentam-se exclusivamente ou predominantemente de anfíbios anuros. Outros itens alimentares importantes são mamíferos (24\%), lagartos (18\%), vertebrados subterrâneos (10\%) e invertebrados (minhocas, moluscos e artrópodes; 15\%). Um total de 51 espécies não tem sido registrado ao longo dos últimos seis anos no município. Provavelmente muitas dessas espécies já estão extintas na região em função da intensa urbanização e perda de hábitats. O levantamento de espécies coletadas no Município de São Paulo e recebidas pelo Instituto Butantan em anos recentes permitiu identificar pelo menos 42 espécies de serpentes e 10 lagartos que ainda ocorrem na região. A elevada riqueza desta fauna pretérita parece ser decorrente da localização geográfica do município, que, no passado, apresentava um mosaico de áreas florestais de Mata Atlântica e formações naturais abertas (cerrados, campos de altitude). Desse modo, a região deveria permitir originalmente a ocorrência concomitante de muitas espécies de áreas florestais e abertas. A fauna de serpentes atual registrada ao longo dos últimos três anos indica que houve uma perda maior da biodiversidade de espécies de formações abertas em relação àquelas de formações florestais.

Palavras-chave: répteis, Município de São Paulo, riqueza, tendências ecológicas.

\section{Introdução}

A América do Sul apresenta grande diversidade de ambientes e abriga uma das herpetofaunas mais ricas do mundo, porém estudos sobre a diversidade de répteis de uma dada localidade ainda são escassos, considerando o tamanho desse continente. Alguns trabalhos listam os répteis de determinada localidade, dando ênfase à caracterização taxonômica das espécies (e.g., Dixon \& Soini 1977, Cunha \& Nascimento 1978). Estudos com esse enfoque, desde que envolvam grande esforço de coleta, permitem caracterizar a riqueza de espécies de uma região. Vários trabalhos abordando a história natural e ecologia de répteis de uma dada localidade foram feitos no Brasil (e.g., Vanzolini 1948, Vitt \& Vangilder 1983, Strüssmann \& Sazima 1993, Sazima \& Haddad 1992, Zimmerman \& Rodrigues 1990, Martins 1991, 1994, França et al. 2006), incluindo a porção sudeste (Sazima \& Haddad 1992, Sazima \& Manzani 1995, Marques \& Sazima 2004, Sawaya et al. 2008). Porém, muitos estudos conduzidos nesta região devem representar subamostragens das espécies que lá ocorrem (q.v. comentários em Vanzolini 1948 e Sazima \& Haddad 1992) e, portanto, não permitem uma boa avaliação da diversidade de espécies. Nessa região, o Município de São Paulo certamente é uma das localidades mais bem amostradas. Registros de répteis na região remontam do século XVI (ver Papavero \& Teixeira 2007), porém a coleta intensiva de material testemunho pela população local iniciou-se há cerca de 100 anos. Além disso, a fauna de serpentes continua sendo bem muito amostrada atualmente em função da presença do Instituto Butantan na cidade de São Paulo.

Nosso objetivo é listar as espécies de répteis registradas no Município de São Paulo ao longo dos últimos 100 anos e caracterizar as tendências ecológicas desta fauna. Além disso, apresentamos a abundância relativa das serpentes registradas no município entre 2003 e 2006. Esses dados são fundamentais para detectar quais espécies de répteis ainda ocorrem em uma região perturbada como o Município de São Paulo e quais são as prováveis necessidades ecológicas de cada espécie (q.v. resultados prévios em Puorto et al. 1991). Este trabalho faz parte de uma série de estudos que estão sendo desenvolvidos sobre a fauna de répteis da área urbana do Município de São Paulo, com ênfase em serpentes. Esses estudos pretendem disponibilizar dados sobre distribuição e história natural das espécies no município (cf. Barbo 2008a,b, Barbo \& Sawaya 2008). Tais informações são essenciais para avaliar as condições locais que permitem a ocorrência desses répteis na região (e.g., grau de preservação de vegetação, tamanho de fragmentos, recursos disponíveis).

\section{Métodos}

Uma quantidade significativa dos espécimes de répteis coletados desde o início do século passado no Município de São Paulo foi tombada nas coleções do Museu de Zoologia da Universidade de São Paulo (MZUSP) e do Instituto Butantan (IBSP). Para elaborar a lista das espécies dessa região consultamos inicialmente os registros de ambas as coleções. Após esse procedimento, todos os exemplares com identificação duvidosa ou questionável foram examinados para sua determinação correta. Incluímos espécies observadas por nós dentro do limite do Município de São Paulo. Excluímos registros de coleção e/ou da literatura com registro único para o Município de São Paulo e com distribuição conhecida distante dessa localidade (>400 km). Um exemplo foi o cágado Mesoclemmys hogei, com registro na literatura para localidade de Rio Pequeno, no Município de São Paulo (SMF 62530). A suposta localidade desse espécime (holótipo) está situada à cerca de $400 \mathrm{~km}$ da ocorrência dos limites dos outros exemplares conhecidos da espécie. Além disso, este exemplar foi obtido no serpentário do Instituto Butantan, onde os registros de procedência podiam ser facilmente confundidos (q.v. também outros argumentos em Rhodin et al. 1982).

Cada espécie foi caracterizada em relação ao bioma de ocorrência e aos seus principais atributos ecológicos, incluindo a utilização de ambiente (área florestal e/ou aberta) e dos recursos associados incluindo o uso de substrato e a dieta. Para determinar os parâmetros ecológicos de cada espécie, foram utilizadas informações prévias obtidas pelos autores deste trabalho, de outros pesquisadores e da literatura (e.g., Marques et al. 2004, Marques 1998, Rodrigues 1990, Ribas \& Monteiro-Filho 2002, Sazima \& Haddad 1992, Sazima \& Abe 1991, Sawaya et al. 2008, Souza 2004, Vanzolini 1948). Ao longo de três anos (agosto de 2003 e julho de 2006) todos exemplares coletados dentro do limite do Município de São Paulo e recebidos pelo IBSP foram tombados na coleção herpetológica denominada MSP, vinculada no momento ao laboratório de Herpetologia do IBSP. Comparamos as tendências ecológicas (uso de ambiente, substrato e dieta) da fauna de répteis atual (2003-2006) e pretérita (últimos 100 anos). A comparação (usando teste qui-quadrado) foi feita considerando apenas as serpentes, uma vez que é o único grupo que pode ser considerado bem amostrado nos últimos anos em função do Instituto Butantan, que recebe grande quantidade desses animais da população de todo o município.

\section{1. Área de estudo}

O Município de São Paulo (Figura 1) apresenta área total de 62.356 ha, dos quais 35.950 ha $(57,7 \%)$ correspondem a áreas urba- 
nizadas e alteradas onde vive cerca de $65 \%$ da população, estimada em 10 milhões de habitantes (Prefeitura do Município de São Paulo 2002). Entre estas áreas urbanizadas e alteradas, apenas 16,4\% (10.226 ha) são ocupados por áreas verdes, sendo 10,2\% (6.360 ha) na zona urbana e $6,1 \%$ (3.803 ha) na zona rural (Silva 1993).

\section{Vegetação}

Mesmo antes do descobrimento, a região do Planalto Paulistano já era um núcleo de povoamento importante ocupado por índios tupiniquins, que provavelmente já modificavam a vegetação original
(Petrone 1995). Um dos primeiros trabalhos sobre a vegetação do Município de São Paulo (Usteri 1911) já relata a ausência de Mata Atlântica primária na região. Porém, não existem dados precisos sobre a vegetação original do Município de São Paulo. Acredita-se que a região apresentava uma paisagem original predominantemente florestal, com áreas de várzea e campos de altitude (Usteri 1911) (Figura 2), eventualmente com a presença de araucária (Araucaria angustifolia) e ocorrência de Cerrado em campos confinados a áreas com condições de solo particulares (Ab'Saber 1963, 1970). O Parque Estadual do Juquery, em Franco da Rocha, dominado por Cerrado,

$47^{\circ} \mathrm{W}$

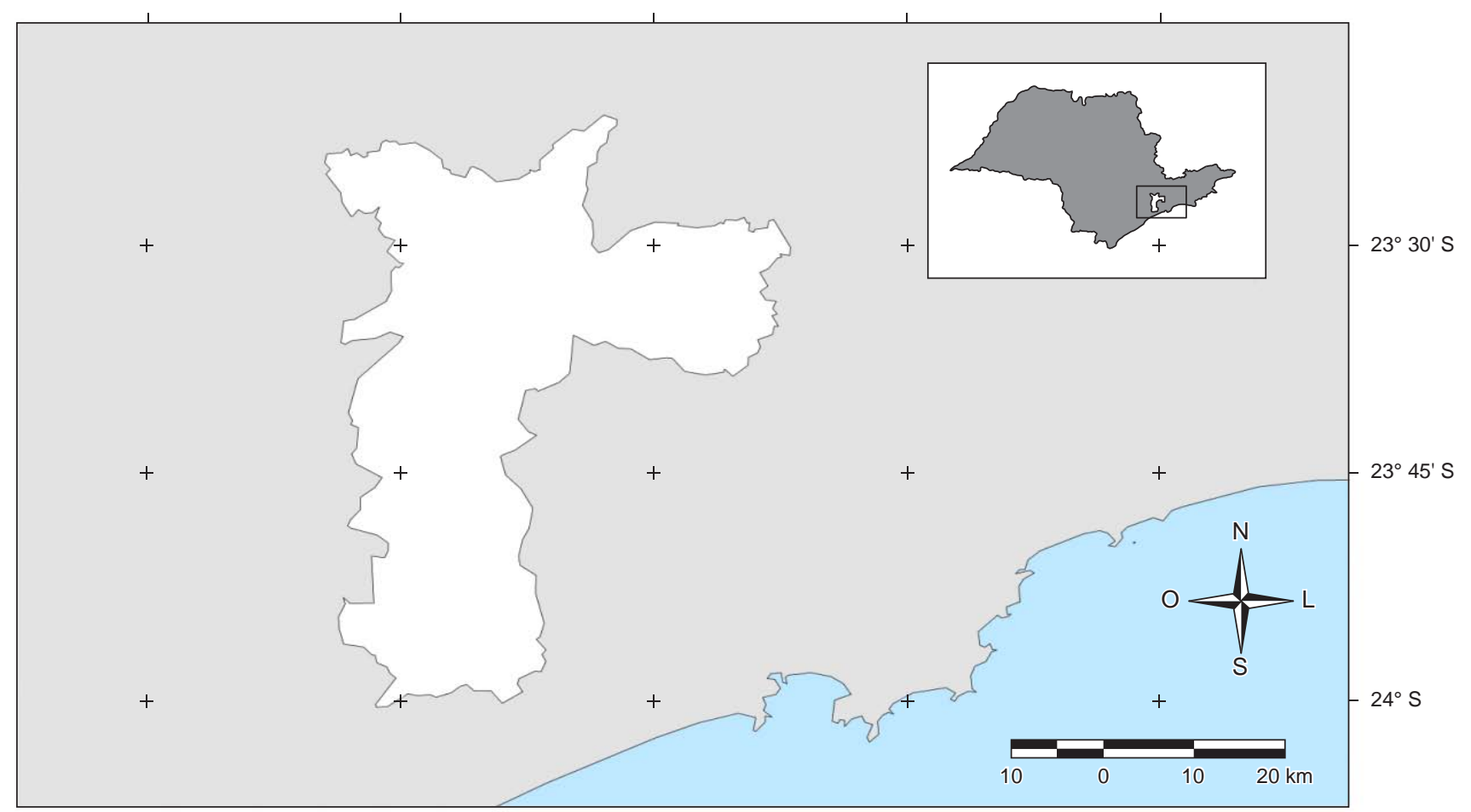

Figura 1. Localização e limites do Município de São Paulo.

Figure 1. Localization and range of the municipality of São Paulo.
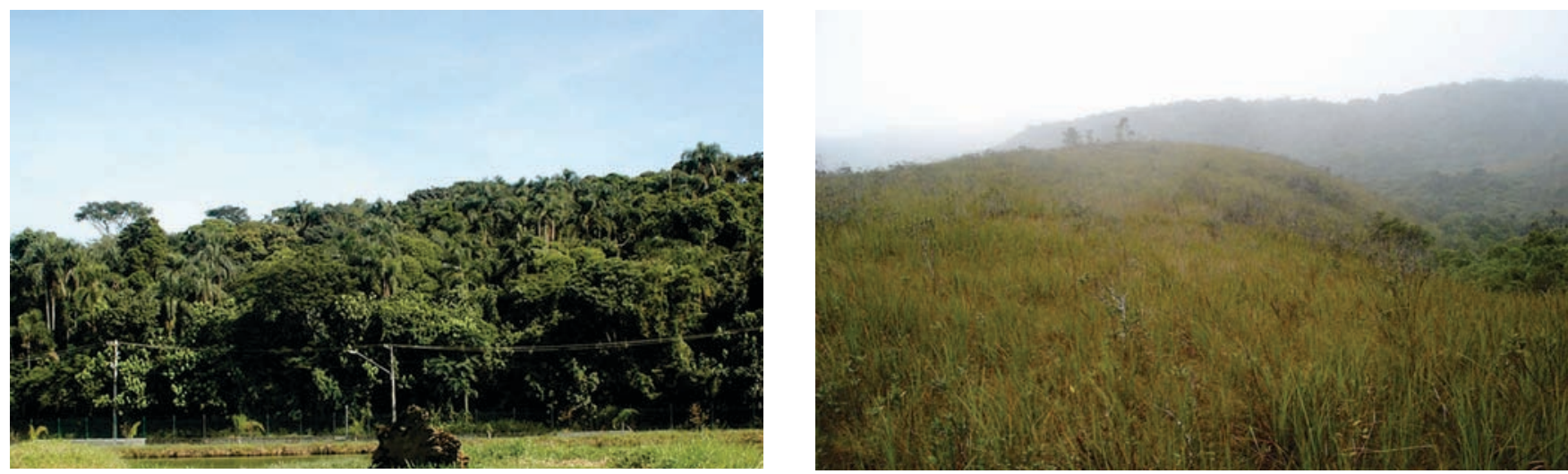

Figura 2. Duas fisionomias ainda presentes e dominantes na paisagem pretérita do Município de São Paulo: Floresta semi-decidual, esquerda (Parque da Estadual Fontes do Ipiranga, zona sul); campo de altitude, direita (Núcleo do Curucutu do Parque Estadual da Serra do Mar, zona sul).

Figure 2. Two current physiognomies which were dominant in the past landscape of the municipality of São Paulo: semi deciduous forest, left (Parque da Estadual Fontes do Ipiranga, south zone); high-altitude grassland right (Núcleo do Curucutu do Parque Estadual da Serra do Mar, south zone). 
está muito próximo do limite noroeste do Município de São Paulo, indicando que originalmente havia manchas naturais deste tipo de ambiente na região.

Atualmente a vegetação é constituída basicamente por fragmentos de vegetação secundária, que ainda resistem ao processo de expansão urbana (Mantovani 2000) (Figura 2). Atualmente existem cerca de 700 fragmentos no Município de São Paulo. A maioria (65\%) possui áreas inferiores a 10 ha e poucos $(3 \%)$ possuem extensão superior a 200 ha. Ao longo do município existem sete unidades de conservação que correspondem a 7.733 ha de áreas protegidas. Os maiores maciços florestais nativos estão confinados nos limites do município. Ao sul, principalmente na Área de Proteção Ambiental (APA) de Capivari - Monos (na península do Bororé) e na margem direita da Represa Guarapiranga, se destacam as formações de floresta ombrófila densa e mata nebular, além dos campos naturais e formações de várzea. Ao norte, nos Parques Estaduais do Jaraguá e da Cantareira e no Parque
Municipal do Anhangüera, incluem significativos remanescentes de floresta ombrófila e campos de altitude (Prefeitura do Município de São Paulo 2002). O extremo leste do município, abriga as APAs do Carmo e Iguatemi, com remanescentes de floresta ombrófila. O Parque Estadual das Fontes do Ipiranga ("Parque do Estado") se destaca por estar totalmente envolvido por área urbana, apresentando-se como uma ilha de vegetação com formações significativas, características de floresta ombrófila densa, floresta estacional e cerrado (Prefeitura do Município de São Paulo 2002).

\section{Resultados}

Foram registradas ao longo dos últimos 100 anos no Município de São Paulo 97 espécies de répteis, sendo duas de quelônios, uma de crocodiliano, 19 de lagartos, sete de anfisbenídeos e 68 de serpentes (Tabela 1).

Tabela 1. Répteis registrados no Município de São Paulo, principal bioma em que ocorrem $(\mathrm{CE}=\mathrm{Cerrado}$ e MA = Mata Atlântica) e atributos ecológicos: uso do ambiente $(\mathrm{A}=$ área aberta e $\mathrm{F}=$ área florestada); uso do substrato $(\mathrm{SU}=$ subterrâneo, $\mathrm{TE}=$ terrícola, $\mathrm{AR}=$ arborícola; $\mathrm{AQ}=\mathrm{aquático})$; e dieta $(\mathrm{ve}=$ vegetais, $\mathrm{mo}=$ molusco, ol = oligoquetos, ar = artrópodes, $\mathrm{pe}=$ peixes, an $=$ anfíbios anuros, ca = cecilídeos ou anfisbenídeos, se $=$ serpentes, la = lagartos, av $=$ aves, $\mathrm{ma}=$ mamíferos, $\mathrm{vr}=$ vertebrados. Asteriscos indicam espécies registradas entre 2003-2006 (dados da recepção do Instituto Butantan e observação pessoal do autores).

Table 1. Reptiles recorded in São Paulo municipality, the main biome of occurrence (CE = Cerrado e MA = Atlantic Forest) and ecological atributes:habitat use $(\mathrm{A}=$ open area e $\mathrm{F}=$ forested area $)$; substrate use $(\mathrm{SU}=$ subterranean, $\mathrm{TE}=$ terrestrial, $\mathrm{AR}=$ arboreal; $\mathrm{AQ}=\mathrm{aquatic})$; and diet $(\mathrm{ve}=\mathrm{vegetables}, \mathrm{mo}=$ mollusks, ol = earthworms, ar = arthropods, $\mathrm{pe}=$ fishes, an $=$ anuran amphibians, $\mathrm{ca}=$ caecilids or amphisbenids, se $=$ snakes, la $=$ lizards, av $=$ birds, $\mathrm{ma}=$ mammals, $\mathrm{vr}=$ vertebrates. Parentheses indicate species recorded between 2003-2006 (data from reception of Instituto Butantan and personal observations of authors).

\begin{tabular}{|c|c|c|c|c|}
\hline & Bioma & Hábitat & Substrato & Dieta \\
\hline \multicolumn{5}{|l|}{ Quelonia } \\
\hline \multicolumn{5}{|l|}{ CHELIDAE } \\
\hline Acanthochelys spixii & $\mathrm{CE}, \mathrm{MA}$ & A & AQ & ar \\
\hline Hydromedusa tectifera & MA & $\mathrm{F}$ & AQ & ar, vr \\
\hline \multicolumn{5}{|l|}{ Crocodila } \\
\hline \multicolumn{5}{|l|}{ ALLIGATORIDAE } \\
\hline * Caiman latirostris ${ }^{1}$ & MA & $\mathrm{F}$ & AQ & $\mathrm{vr}$ \\
\hline \multicolumn{5}{|l|}{ Lacertilia } \\
\hline \multicolumn{5}{|l|}{ GEKONIDAE } \\
\hline *Hemidactylus mabouia ${ }^{2}$ & CE, MA & $\mathrm{A}, \mathrm{F}$ & $\mathrm{AR}$ & ar \\
\hline \multicolumn{5}{|l|}{ POLYCHRIDAE } \\
\hline Anisolepis grilii & $\mathrm{MA}^{(\mathrm{pl})}$ & $\mathrm{F}$ & $\mathrm{AR}$ & ar \\
\hline Enyalius iheringii & MA & $\mathrm{F}$ & $\mathrm{AR}$ & ar \\
\hline Enyalius perditus & MA & $\mathrm{F}$ & $\mathrm{AR}$ & ar \\
\hline Polychrus acutirostris & $\mathrm{CE}$ & $\mathrm{A}$ & AR & ar \\
\hline Urostrophus vautieri & $\mathrm{MA}^{(\mathrm{pl})}$ & $\mathrm{F}$ & AR & ar \\
\hline \multicolumn{5}{|l|}{ GYMNOPHTALMIDAE } \\
\hline Colobodactylus taunayi & MA & $\mathrm{F}$ & $\mathrm{TE}$ & ar \\
\hline Ecpleopus gaudichaudii & MA & $\mathrm{F}$ & $\mathrm{TE}$ & ar \\
\hline Heterodactylus imbricatus & MA & $\mathrm{F}$ & $\mathrm{TE}$ & ar \\
\hline Pantodactylus quadrilineatus & MA & $\mathrm{F}$ & TE & ar \\
\hline Pantodactylus schreibersii & MA & $\mathrm{F}$ & $\mathrm{TE}$ & ar \\
\hline Placosoma glabelum & MA & $\mathrm{F}$ & $\mathrm{AR}$ & ar \\
\hline \multicolumn{5}{|l|}{ TEIIDAE } \\
\hline *Ameiva ameiva & $\mathrm{CE}$ & A & $\mathrm{TE}$ & ar \\
\hline * Tupinambis merianae & MA & $\mathrm{F}$ & $\mathrm{TE}$ & ar, vr, ve \\
\hline \multicolumn{5}{|l|}{ TROPIDURIDAE } \\
\hline *Tropidurus itambere & $\mathrm{CE}$ & A & TE & ar \\
\hline
\end{tabular}


Répteis do Município de São Paulo

Tabela 1. Continuação...

\begin{tabular}{|c|c|c|c|c|}
\hline & Bioma & Hábitat & Substrato & Dieta \\
\hline \multicolumn{5}{|l|}{ ANGUIDAE } \\
\hline Ophiodes fragilis & MA & $\mathrm{F}$ & TE & ar \\
\hline Ophiodes striatus & $\mathrm{CE}$ & A & $\mathrm{TE}$ & ar \\
\hline \multicolumn{5}{|l|}{ SCINCIDAE } \\
\hline Mabuya dorsivittata & $\mathrm{CE}$ & A & TE, ar & ar \\
\hline Mabuya frenata & $\mathrm{CE}$ & $\mathrm{F}$ & TE, ar & ar \\
\hline \multicolumn{5}{|l|}{ Amphisbaenia } \\
\hline \multicolumn{5}{|l|}{ AMPHISBAENIDAE } \\
\hline *Amphisbaena alba & $\mathrm{CE}$ & A & SU & ar \\
\hline *Amphisbaena dubia & $\mathrm{CE}$ & A & SU & ar \\
\hline Amphisbaena hogei & MA & $\mathrm{F}$ & SU & ar \\
\hline *Amphisbaena mertensi & $\mathrm{CE}$ & A & SU & ar \\
\hline *Amphisbaena trachura & $\mathrm{CE}$ & A & SU & ar \\
\hline Cercolophia roberti & $\mathrm{MA}^{(\mathrm{pl})}$ & A & SU & ar \\
\hline *Leposternon microcephalum & MA & $\mathrm{F}$ & SU & ol, ar \\
\hline \multicolumn{5}{|l|}{ Serpentes } \\
\hline \multicolumn{5}{|l|}{ ANOMALEPIDIDAE } \\
\hline *Liotyphlops beui & $\mathrm{MA}^{(\mathrm{pl})}$ & $\mathrm{F}$ & SU & ar \\
\hline \multicolumn{5}{|l|}{ TROPIDOPHIIDAE } \\
\hline *Tropidophis paucisquamis & $\mathrm{MA}^{(\mathrm{pl})}$ & $\mathrm{F}$ & $\mathrm{AR}$ & an \\
\hline \multicolumn{5}{|l|}{ COLUBRIDAE } \\
\hline *Apostolepis assimilis & $\mathrm{CE}$ & A & SU & $\mathrm{ca}$ \\
\hline Apostolepis dimidiata & $\mathrm{CE}$ & A & SU & $\mathrm{ca}$ \\
\hline *Atractus pantostictus & $\mathrm{CE}, \mathrm{MA}^{(\mathrm{pl})}$ & $\mathrm{F}$ & SU & ol \\
\hline *Atractus reticulatus & $\mathrm{CE}, \mathrm{MA}^{(\mathrm{pl})}$ & $\mathrm{F}$ & SU & ol \\
\hline *Atractus zebrinus & MA & $\mathrm{F}$ & SU & ol \\
\hline Boiruna maculata & $\mathrm{CE}$ & A & TE & se, la, ma \\
\hline *Chironius bicarinatus & MA & $\mathrm{F}$ & $\mathrm{AR}$ & an \\
\hline *Chironius exoletus & MA & $\mathrm{F}$ & $\mathrm{AR}$ & an \\
\hline Chironius flavolineatus & $\mathrm{CE}$ & $\mathrm{A}, \mathrm{F}$ & $\mathrm{AR}$ & an \\
\hline Chironius quadricarinatus & $\mathrm{CE}$ & $\mathrm{A}, \mathrm{F}$ & $\mathrm{AR}$ & an \\
\hline Clelia plumbea & MA & $\mathrm{F}$ & TE & se, la, ma \\
\hline *Clelia quimi & $\mathrm{CE}, \mathrm{MA}$ & $\mathrm{A}, \mathrm{F}$ & TE & ma, la, se \\
\hline Echinanthera amoena & MA & $\mathrm{F}$ & TE & an \\
\hline *Echinanthera cephalostriata & MA & $\mathrm{F}$ & TE & an \\
\hline Echinanthera cyanopleura & MA & $\mathrm{F}$ & TE & an \\
\hline *Echinanthera undulata & MA & $\mathrm{F}$ & TE & an \\
\hline Elapomorphus quinquelineatus & MA & $\mathrm{F}$ & SU & $\mathrm{ca}$ \\
\hline *Erythrolamprus aesculapii & $\mathrm{CE}, \mathrm{MA}$ & $\mathrm{F}, \mathrm{A}$ & TE & se \\
\hline Gomesophis brasiliensis & MA & $\mathrm{F}$ & AQ & ol \\
\hline *Helicops carinicaudus & MA & $\mathrm{F}$ & AQ & pe \\
\hline *Helicops modestus & $\mathrm{CE}$ & A & AQ & pe \\
\hline Liophis atraventer & MA & $\mathrm{F}$ & $\mathrm{TE}$ & an \\
\hline Liophis almadensis & $\mathrm{CE}$ & A & $\mathrm{AQ}, \mathrm{TE}$ & an \\
\hline Liophis flavifrenatus & $\mathrm{CE}$ & A & $\mathrm{TE}$ & an \\
\hline *Liophis miliaris & MA & $\mathrm{F}$ & $\mathrm{AQ}, \mathrm{TE}$ & an, pe \\
\hline *Liophis jaegeri & CE, MA & $\mathrm{A}, \mathrm{F}$ & $\mathrm{TE}$ & an \\
\hline *Liophis poecilogyrus & $\mathrm{CE}$ & $\mathrm{A}, \mathrm{F}$ & $\mathrm{TE}$ & an \\
\hline Liophis reginae & $\mathrm{CE}$ & A & $\mathrm{TE}$ & an \\
\hline *Liophis typhlus & MA & $\mathrm{F}$ & $\mathrm{TE}$ & an \\
\hline Mastigodryas bifossatus & $\mathrm{CE}$ & A & $\mathrm{TE}$ & an, ma \\
\hline
\end{tabular}


Tabela 1. Continuação...

\begin{tabular}{|c|c|c|c|c|}
\hline & Bioma & Hábitat & Substrato & Dieta \\
\hline *Oxyrhopus clathratus & $\mathrm{MA}^{(\mathrm{pl})}$ & $\mathrm{F}$ & $\mathrm{TE}$ & ma, la \\
\hline *Oxyrhopus guibei & $\mathrm{CE}$ & $\mathrm{A}, \mathrm{F}$ & $\mathrm{TE}$ & ma, la \\
\hline Phalotris mertensi & $\mathrm{CE}$ & A & SU & $\mathrm{ca}$ \\
\hline Philodryas aestivus & $\mathrm{CE}$ & A & AR, TE & an, ma \\
\hline *Philodryas olfersii & CE, MA & $\mathrm{F}$ & AR, TE & an, ma \\
\hline Philodryas mattogrossensis & $\mathrm{CE}$ & $\mathrm{F}$ & $\mathrm{AR}, \mathrm{TE}$ & an, ma \\
\hline *Philodryas patagoniensis & CE, MA & A & $\mathrm{AR}, \mathrm{TE}$ & an, ma \\
\hline Phimophis guerini & $\mathrm{CE}$ & A & $\mathrm{TE}$ & la \\
\hline Pseudoboa serrana & $\mathrm{MA}^{(\mathrm{pl})}$ & $\mathrm{F}$ & $\mathrm{TE}$ & la, ma \\
\hline *Sibynomorphus mikanii & $\mathrm{CE}, \mathrm{MA}^{(\mathrm{pl})}$ & $\mathrm{A}, \mathrm{F}$ & $\mathrm{TE}$ & mo \\
\hline *Sibynomorphus neuwiedii & MA & $\mathrm{F}$ & TE, AR & mo \\
\hline Simophis rhinostoma & $\mathrm{CE}$ & A & TE & an \\
\hline *Siphlophis longicaudatus & $\mathrm{MA}^{(\mathrm{pl})}$ & $\mathrm{F}$ & $\mathrm{AR}$ & la \\
\hline Siphlophis pulcher & MA & $\mathrm{F}$ & $\mathrm{AR}$ & la \\
\hline Sordellina punctata & MA & $\mathrm{F}$ & AQ & ol \\
\hline *Spilotes pullatus & MA & $\mathrm{F}$ & $\mathrm{AR}, \mathrm{TE}$ & ma, av \\
\hline *Taeniophallus affinis & MA & $\mathrm{F}$ & $\mathrm{CR}$ & an \\
\hline Taeniophallus bilineatus & MA & $\mathrm{F}$ & $\mathrm{CR}$ & an \\
\hline Taeniophallus occipitalis & $\mathrm{CE}$ & A & $\mathrm{CR}$ & an \\
\hline Taeniophallus persimilis & MA & $\mathrm{F}$ & CR & an \\
\hline *Tantilla melanocephala & $\mathrm{CE}$ & A & $\mathrm{CR}$ & ar \\
\hline Thamnodynastes cf. nattereri & MA & $\mathrm{F}$ & TE, AR & an \\
\hline Thamnodynastes hypoconia & CE, MA & $\mathrm{A}, \mathrm{F}$ & TE, AR & an, la \\
\hline *Thamnodynastes strigatus & CE, MA & $\mathrm{A}, \mathrm{F}$ & TE, AR & an, ma \\
\hline *Tomodon dorsatus & MA & $\mathrm{F}$ & TE & mo \\
\hline Tropidodryas serra & MA & $\mathrm{F}$ & TE, AR & ma, la \\
\hline *Tropidodryas striaticeps & $\mathrm{MA}^{(\mathrm{pl})}$ & $\mathrm{F}$ & TE, AR & ma, la \\
\hline Uromacerina ricadinii & MA & $\mathrm{F}$ & $\mathrm{AR}$ & an \\
\hline Waglerophis merremii & $\mathrm{CE}$ & A & $\mathrm{TE}$ & an \\
\hline *Xenodon neuwiedii & MA & $\mathrm{F}$ & $\mathrm{TE}$ & an \\
\hline \multicolumn{5}{|l|}{ ELAPIDAE } \\
\hline Micrurus decoratus & $\mathrm{MA}^{(\mathrm{pl})}$ & $\mathrm{F}$ & SU & $\mathrm{ca}$ \\
\hline *Micrurus corallinus & MA & $\mathrm{F}$ & SU & $\mathrm{ca}$ \\
\hline Micrurus frontalis & $\mathrm{CE}$ & $\mathrm{A}, \mathrm{F}$ & SU & $\mathrm{ca}$ \\
\hline *Micrurus lemniscatus & MA & $\mathrm{A}, \mathrm{F}$ & SU & $\mathrm{ca}$ \\
\hline \multicolumn{5}{|l|}{ VIPERIDAE } \\
\hline *Bothrops jararaca & MA & $\mathrm{F}$ & $\mathrm{TE}$ & ma, an \\
\hline *Crotalus durissus & $\mathrm{CE}$ & $\mathrm{A}$ & TE & $\mathrm{ma}$ \\
\hline
\end{tabular}

(pl) Encontrada exclusiva ou predominantemente na Mata Atlântica de planalto, geralmente em altitudes > 600 m, 1-sem espécimes testemunho em coleção, mas existem relatos de exemplares nos rios Tietê e Pinheiros dentro dos limites do município, 2-espécie exótica, 3-inclui vertebrados.

Aproximadamente $60 \%$ das espécies registradas ocorrem usualmente na Mata Atlântica da Serra do Mar e as demais são registradas em áreas abertas do domínio de Cerrado no interior do estado. Algumas espécies também podem ser encontradas nas duas regiões indistintamente. A maioria dos répteis registrados vive em áreas florestadas, e mesmo entre as espécies mais típicas de Cerrado, algumas vivem associadas às áreas florestadas, como matas de galeria (e.g., Atractus pantostictus) (cf. Sawaya et al. 2008).

Todas as tartarugas e o jacaré são típicos de ambientes aquáticos e possuem dieta baseada em peixes e invertebrados aquáticos. Entre os lagartos há espécies arborícolas (32\%) e terrícolas (68\%) e quase todos dependem de artrópodes para sua alimentação (ver Tabela 1).
A maior parte das serpentes possui hábitos exclusivamente terrícolas (38\%), as demais usam substratos subterrâneos ou sob a serapilheira (25\%), sobre a vegetação (18\%) ou vivem associadas a corpos d'água (9\%). Praticamente metade das serpentes alimenta-se exclusivamente ou predominantemente de anfíbios anuros e cerca de um quarto (24\%) utilizam mamíferos. Outros itens alimentares incluem lagartos (18\%), vertebrados subterrâneos (10\%), e invertebrados (minhocas, moluscos e artrópodes; $15 \%$ ).

Entre agosto de 2003 e julho de 2006 foram recebidas pelo Instituto Butantan 1370 serpentes de 37 espécies (Figura 3). As espécies mais frequientes foram Oxyrhopus guibei (25\% do número total de 


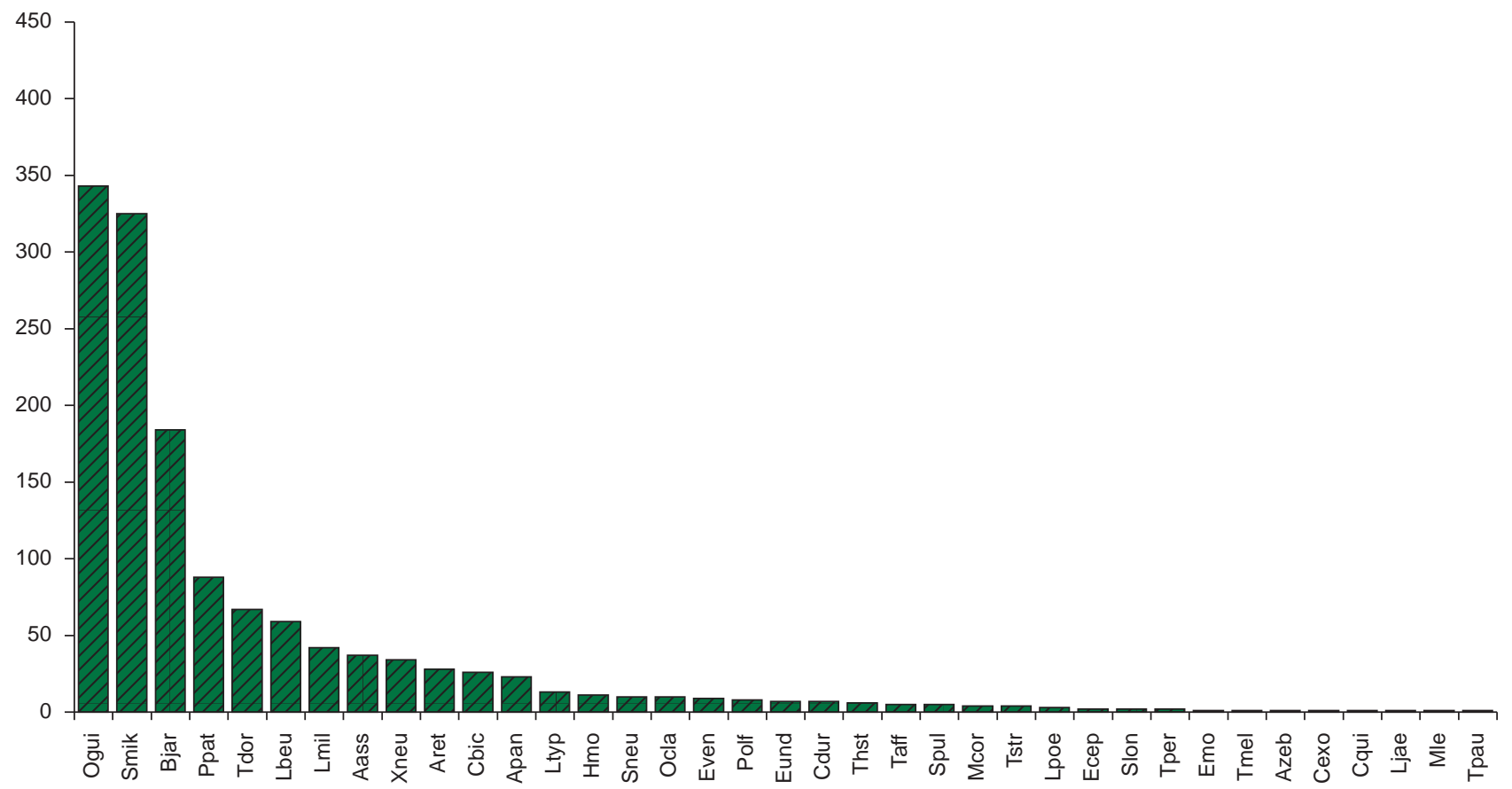

Figura 3. Abundância relativa das serpentes do Município de São Paulo trazidas ao Instituto Butantan entre agosto de 2003 e julho de 2006 ( $\mathrm{N}=1372$ ). Legenda: Aass = Apostolepis assimilis; Apan = Atractus pantostictus; Aret = Atractus reticulatus; Azeb = Atractus zebrinus; Bjar = Bothrops jararaca $;$ Cbic = Chironius bicarinatus $;$ Cexo = Chironius exoletus $;$ Cqui $=$ Clelia quimi $;$ Cdur $=$ Crotalus durissus $;$ Emon = Erythrolamprus aesculapii monozona $;$ Even $=$ Eryth rolamprus a. venustissimus; Ecep = Echinanthera cephalostriata $;$ Eund = Echinanthera undulata $;$ Hcar = Helicops carinicaudus; Hmod = Helicops modestus; Lbeu $=$ Liotyphlops beui $;$ Ljae $=$ Liophis jaegeri $;$ Lmil = Liophis miliaris $;$ Lpoe = Liophis poecilogyrus $;$ Ltyp $=$ Liophis typhlus $;$ Mcor $=$ Micrurus corallinus; Mlem = Micrurus lemniscatus; Ocla = Oxyrhopus clathratus; Ogui = Oxyrhopus guibe $;$ Polf $=$ Philodryas olfersii Ppat $=$ Philodryas patagoniensis $;$ Slon $=$ Siphlophis longicaudatus; Smik = Sibynomorphus mikanii; Sneu = Sibynomorphus neuwiedi $;$ Spul = Spilotes pullatus; Taff = Taeniophallus affinis; Tper $=$ Taeniophallus persimilis; Tmel = Tantilla melanocephala $;$ Thst = Thamnodynastes strigatus; Tdor = Tomodon dorsatus; Tpau = Tropidophis paucisquamis; Tstr $=$ Tropidodryas striaticeps; Xneu = Xenodon neuwiedii .

Figure 3. Relative abundance of the snakes of São Paulo municipality brought by lay people to the Instituto Butantan between August of 2003 and July of $2006(\mathrm{~N}=1372)$. Legend: Aass = Apostolepis assimilis; Apan = Atractus pantostictus $;$ Aret = Atractus reticulatus; Azeb = Atractus zebrinus; Bjar = Bothrops jararaca $;$ Cbic $=$ Chironius bicarinatus $;$ Cexo $=$ Chironius exoletus $;$ Cqui $=$ Clelia quimi $;$ Cdur $=$ Crotalus durissus; Emon = Erythrolamprus aesculapii monozona $;$ Even = Erythrolamprusa. venustissimus; Ecep = Echinanthera cephalostriata $;$ Eund = Echinanthera undulata $;$ Hcar = Helicops carinicaudus; Hmod = Helicops modestus; Lbeu = Liotyphlops beui $;$ Ljae = Liophis jaegeri $;$ Lmil = Liophis miliaris; Lpoe = Liophis poecilogyrus; Ltyp = Liophis typhlus; Mcor = Micrurus corallinus; Mlem = Micrurus lemniscatus $;$ Ocla = Oxyrhopus clathratus $;$ Ogui $=$ Oxyrhopus guibei $;$ Polf $=$ Philodryas olfersii $;$ Ppat $=$ Philodryas patagoniensis $;$ Slon = Siphlophis longicaudatus $;$ Smik = Sibynomorphus mikanii $;$ Sneu = Sibynomorphus neuwiedi $;$ Spul = Spilotes pullatus $;$ Taff = Taeniophal lus affinis $;$ Tper $=$ Taeniophallus persimilis $;$ Tmel $=$ Tantilla melanocephala $;$ Thst $=$ Thamnodynastes strigatus $;$ Tdor $=$ Tomodon dorsatus $;$ Tpau $=$ Tropidophis paucisquamis; Tstr = Tropidodryas striaticeps; Xneu = Xenodon neuwiedii.

indivíduos), Sibynomorphus mikanii (24\%) e Bothrops jararaca (13\%). Outras espécies relativamente comuns foram Philodryas patagoniensis (6\%), Tomodon dorsatus (5\%) e Liotyphlops beui (4\%) (Figura 2). As tendências ecológicas da fauna atual (registrada entre 2003 e 2006) em relação proporção de espécies que utilizam determinado recurso (alimento e substrato), são semelhantes às da fauna de serpentes registrada últimos 100 anos. Entretanto, a fauna pretérita apresentava número de espécies de áreas abertas significativamente maior do que a atual $\left(\chi^{2}=7,35, \mathrm{p}<0,01\right.$; ver Figura 4); mas tal diferença não foi significativa quando consideradas espécies que usam áreas florestais ou ambas as áreas (abertas/florestais) $\left(\chi^{2}=3,16, p=0,08\right.$ para áreas florestais e $\chi^{2}=0,80, p=0,37$, para ambas áreas; Figura 4).

\section{Discussão}

O Município de São Paulo (MSP) está inserido no domínio da Mata Atlântica e a riqueza de répteis parece ser bastante elevada quando comparada a outras regiões dentro desse domínio. Em parte essa elevada riqueza de espécies do MSP deve-se ao fato dessa área ter sido muita bem amostrada. A presença do Instituto Butantan e do
Museu de Zoologia na região desde o início do século XX certamente garantiram essa boa amostragem ao longo dos últimos 100 anos. Porém, outras áreas dentro do domínio da Mata Atlântica também foram bem amostradas e apresentam riqueza menor de espécies. Não estão disponíveis estimativas precisas da riqueza da fauna de répteis em regiões de Mata Atlântica no Planalto Paulistano, devido a deficiências de amostragem (e.g., Serra do Japi, cf. Haddad \& Sazima 1992) ou ausência de compilação de dados, pois há regiões bem amostradas com material disponível nas coleções MZUSP e IBSP. Entretanto, os dados sobre regiões de Mata Atlântica na baixada litorânea indicam riqueza bem menor. A Estação Ecológica Juréia-Itatins, litoral sul de São Paulo, com área de 80.000 ha, possui 36 espécies de répteis registrados. Quando acrescentados dados de municípios nos arredores incluindo áreas de maior altitude, esse número atinge cerca de 50 espécies (cf. Marques \& Sazima 2004). Portanto, parece existir praticamente o dobro de espécies de répteis no Município de São Paulo (ca 60.000 ha) em relação à região de Juréia-Itatins (80.000 ha). A alta riqueza de répteis do Município de São Paulo também é evidente quando consideradas somente as serpentes. A Serra do Mar, que se estende em uma faixa desde Santa Catarina até o Rio de Janeiro, possui 80 espécies de serpentes (Marques et al. 2004), ao passo que 


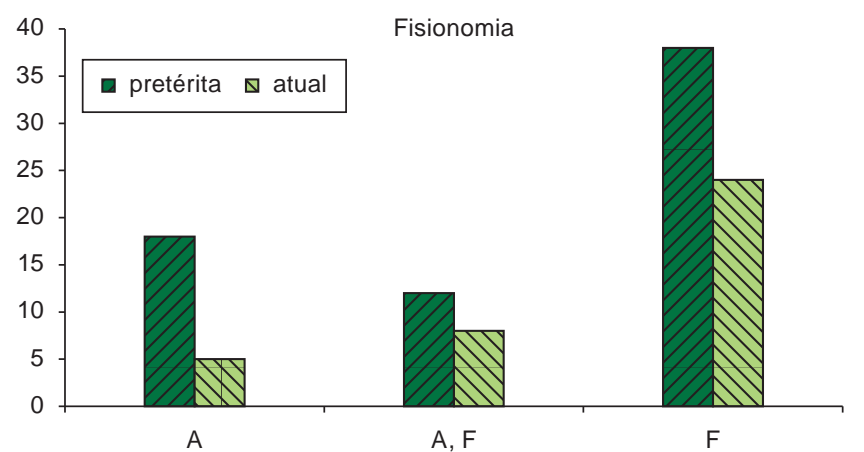

Figura 4. Número de espécies de serpentes que ocorrem em áreas abertas (A) e florestadas $(\mathrm{F})$ ou em ambas $(\mathrm{A}, \mathrm{F})$. A fauna pretérita corresponde a lista elaborada por consulta à coleções e a atual aos animais coletados entre agosto de 2003 e julho de 2006.

Figure 4. Number of snake species recorded in open areas (A) and forests areas $(\mathrm{F})$ or in both $(\mathrm{A}, \mathrm{F})$, in the municipality of São Paulo. The past fauna was obtained through the records in Herpetological Collections, and data on extant fauna is based in snakes brought by lay people to the Instituto Butantan between August of 2003 and July of 2006.

no MSP foram registradas aproximadamente 70 espécies (presente trabalho). Portanto, a riqueza de espécies registradas para o Município de São Paulo é de fato bastante elevada, sendo comparável inclusive com àquelas de regiões estudadas do Cerrado (e.g., Parque Nacional das Emas, GO, 86 espécies em 130.000 ha, Valdujo et al. em prep.; Região do Manso, MT, > 40 espécies, C. Strüssmann, com. pess..) e da Amazônia (e.g., Zimmerman \& Rodrigues 1990, Martins \& Oliveira 1999, Dixon \& Soini 1977).

O Município de São Paulo está localizado no Planalto Atlântico próximo a Serra do Mar (Almeida 1964) em região originalmente recoberta por vegetação de várzea, campos e principalmente florestas (Usteri 1911). A existência de um mosaico ambiental no Município de São Paulo com predominância de áreas florestais, mas com várias porções de fisionomias abertas permitiu a ocorrência concomitante de espécies com exigências ecológicas distintas. De fato, a fauna de répteis registrada para o Município de São Paulo inclui diversas espécies características de áreas florestais e outras típicas de áreas abertas (Barbo 2008a,b, Barbo \& Sawaya 2008). Provavelmente, isto se deve ao fato de ter existido em boa parte do município, nas primeiras décadas do século passado, um mosaico de áreas florestais (Mata Atlântica) e de formações abertas (e.g., campos de várzea e de altitude e Cerrado) (Usteri 1911). A região abriga várias espécies típicas das florestas úmidas da vertente oceânica da Serra do Mar (e.g., Enyalius iheringhi, Tropidodryas serra, Micrurus corallinus, Tropidophis paucisquamis), mas outras comuns em florestas semideciduais do planalto (e.g., Urostrophus vautieri). Há registros de espécies que ocorrem usualmente na baixada litorânea (e.g., Enyalius iheringi, Placosoma glabelum, Chironius bicarinatus) e outras de típicas de altitudes superiores a $600 \mathrm{~m}$ (e.g., Atractus zebrinus, Pseudoboa serrana, Micrurus decoratus). Portanto, a heterogeneidade ambiental, que inclui áreas abertas e formações vegetais distintas com altitude variável, parece ser fator determinante da elevada riqueza local de espécies.

Provavelmente, uma parcela significativa das espécies conhecidas para a região já foi extinta localmente devido à intensa urbanização local, (cf. Puorto et al. 1991) e a perda de $84 \%$ dos hábitats originais locais. Flutuações naturais da composição de répteis podem ocorrer e o curto período de amostragem da fauna atual (2003-2006) impossibilitam afirmar com segurança quais espécies devem ter desaparecido do Município de São Paulo. Porém, a amostra de serpentes entre o período de 2003-2006 é grande $(n=1370)$ e deve refletir a riqueza atual de serpentes (q.v. Barbo 2008a para estimativas de riqueza em diferentes regiões do município), indicando que poucas espécies serão acrescentadas nesta lista e que praticamente metade das espécies que ocorriam originalmente na região foram extintas.

Além das extinções, outras espécies (e.g., Crotalus durissus) podem ter invadido e colonizado algumas áreas em função das alterações ocorridas nas fisionomias nativas (cf. Marques et al. 1998, 2004). Mais de metade das serpentes encontradas atualmente no município pertencem a 3 espécies. As duas mais comuns (Oxyrhopus guibei e Sibynomorphus mikanii) são freqüentemente encontradas dentro da região urbana. A terceira (Bothrops jararaca) se restringe às áreas mais preservadas de mata nas zonas sul e norte do município e aos parques do Estado e do Carmo. O modo de coleta das serpentes, baseado em coleta pela população local, deve subamostrar espécies de hábitos subterrâneos (e.g, Liotyphlops beui, Apostolepis assimilis, Atractus reticulatus). Entre as serpentes subterrâneas, Liotyphlops beui foi a mais coletada, embora apresente pequeno porte, tenha hábito estritamente noturno e seja a mais subterrânea entre todas as espécies amostradas (cf. Parpinelli, 2008, Parpinelli \& Marques 2008). Portanto, é possível que a cobra-cega $L$. beui seja, inclusive, a espécie mais abundante do Município de São Paulo. Estudos incluindo a coleta com armadilhas de interceptação e queda ("pitfall traps") poderiam auxiliar na compreensão dessa questão, uma vez que esse método permite amostrar de forma eficiente serpentes de hábitos subterrâneos (Martins \& Oliveira 1999, Sawaya 2004). Entre as serpentes mais frequientes no Município de São Paulo, há espécies noturnas, que predam invertebrados (Liotyphlops beui, Atractus reticulatus e Sibynomorphus mikanii) e roedores (Oxyrhopus guibei e Bothrops jararaca). Tais atributos ecológicos (hábito noturno e dieta baseada em invertebrados ou roedores) parecem possibilitar a subsistência destas serpentes com relativo sucesso em uma área altamente urbanizada como São Paulo (cf. Puorto et al. 1991). Duas dessas serpentes (Liotyphlops beui e Sibynomorphus mikanii) parecem ser abundantes em ambientes muito alterados (e.g., terrenos baldios) (cf. Barbo 2008a,b) e poderiam ser classificadas como sinantrópicas. Entretanto, as demais estão associadas a fragmentos florestais (Barbo 2008a,b). Outras serpentes e lagartos registrados no município dependem da estrutura complexa da vegetação e de recursos alimentares escassos em áreas urbanizadas (e.g., Chironius spp.) e, portanto, são mais sensíveis e dependem de áreas preservadas com vegetação nativa (Marques \& Sazima 2004). Portanto, a manutenção de fragmentos florestais é fundamental para manutenção de grande parte das espécies encontradas no município.

As regiões mais preservadas e conectadas com áreas de preservação de grande extensão (e.g., Serra da Cantareira e Parque do Carmo) devem permitir a existência de maior diversidade, ao passo que áreas isoladas (e.g., Parque do Estado) devem apresentar uma fauna mais depauperada. É necessária uma avaliação mais detalhada dessas áreas para avaliar a composição e diversidade de espécies, assim como quais espécies podem ser mantidas em fragmentos isolados. Ainda existem poucos estudos do efeito da fragmentação sobre a comunidade de répteis (ver Colli 2003, Silvano et al. 2005, Dixo \& Verdade 2006, Faria et al. 2007). Portanto, estudos futuros nos remanescentes naturais do Município de São Paulo poderão ser fundamentais para monitorar e compreender melhor como a fragmentação afeta a comunidade de répteis.

Ainda é difundida a idéia que espécies de áreas abertas sejam em geral menos afetadas por mudanças no hábitat que aquelas vivem em áreas florestais. Acredita-se inclusive que as espécies de áreas abertas poderiam colonizar e até expandir sua ocorrência para ambientes antrópicos. Embora alguns elementos da fauna de répteis de florestas sejam suscetíveis às alterações antrópicas (q.v. Marques \& Sazima 
2004), os dados aqui obtidos não suportam essa visão, uma vez que a maior perda de biodiversidade no município ocorreu entre as espécies associadas á áreas abertas. Tais espécies estão frequientemente associadas ao domínio do Cerrado, que abriga principalmente hábitats abertos, como campos e savanas naturais. Uma vez que a fauna de áreas abertas é mais sensível a alterações ambientais em relação à de áreas florestais é importante reforçar a necessidade e urgência na implementação de medidas de conservação dos campos naturais no município, no estado e em todo o país. Outros estudos já detectaram que algumas espécies de serpentes são dependentes de áreas de campos (Marques et al. 2006) e o mesmo tem sido verificado para espécies de lagartos (Nogueira et al. 2005; Nogueira 2006). Espécies de répteis que vivem no Cerrado estão em geral associadas a manchas de hábitat específicos, sendo os ambientes florestais verdadeiras barreiras à sua dispersão (ver Nogueira et al. 2005, Nogueira et al. 2009). Portanto, é provável que no passado muitas espécies do Município de São Paulo estariam restritas à manchas de áreas abertas já pouco abundantes na paisagem, dominada por florestas (Usteri 1911). Tais manchas hoje praticamente inexistem no município comprometendo de forma significativa a composição e a estrutura da herpetofauna nativa.

\section{Agradecimentos}

Aos colegas Myriam E. Calleffo, H. Ferrarezzi, Fernanda S. Oliveira, Ricardo A. K. Ribeiro, Miguel T. Rodrigues, Murilo Rodrigues e Franco L. Souza que forneceram informações complementares sobre biologia dos répteis, de exemplares tombados em coleções e bibliografia. A Frederico França e Cristiano Nogueira pelas sugestões que enriqueceram o manuscrito. Ao CNPq e FAPESP pelo apoio financeiro.

\section{Referências Bibliográficas}

AB' SÁBER, A.N. 1963. Originalidade do sítio da cidade de São Paulo. Acrópole, 295-296:239-246

AB' SÁBER, A.N. 1970. O mosaico primário de matas e cerrados do Planalto Paulistano. Cadernos de Ciências da Terra, 6:24-26.

BARBO, F.E. 2008a. Composição, História Natural, Diversidade e Distribuição das Serpentes no Município de São Paulo, SP. Dissertação de Mestrado, Universidade de São Paulo, São Paulo.

BARBO, F.E. 2008b. Os Répteis no Município de São Paulo: aspectos históricos, diversidade e conservação. In Além do Concreto: contribuições para a proteção da biodiversidade paulistana (L. R. Malagoli, F.B. Bajestero \& M. Whately, eds). Editora Instituto Socioambiental, São Paulo, p. 234-267.

BARBO, F.E. \& SAWAYA, R.J. 2008. Amphisbaenians, municipality of São Paulo, Southeastern Brazil. Checklist, 4(1):5-11.

COLLI, G.R. 2003. Estrutura de taxocenoses de lagartos em fragmentos naturais e antrópicos de Cerrado. In Ecossistemas Brasileiros: Manejo e Conservação (V. Claudino-Sales, ed). Expressão Gráfica e Editora, Fortaleza, p. 171-178.

CUNHA, O.R. \& NASCIMENTO, F.P. 1978. Ofídios da Amazônia X. As cobras da região leste do Pará, Belém. Mus. Par. Emílio Goeldi Publ. Avulsas, 31(1):7-166.

DIXON, J.R. \& SOINI, P. 1977. The reptiles of the upper Amazon basin, Iquitos region, Peru. II. Crocodilians, turtles, and snakes. Milwakee Publ. Mus. Contr. Biol. Geol. 12:1-71.

DIXO, M. \& VERDADE, K.V. 2006. Herpetofauna de serrapilheira da Reserva Florestal de Morro Grande, Cotia (SP). Biota Neotropica, 6(2):1-20.

FARIA, D., PACIENCIA, M.L.B., DIXO, M., LAPS, R.R. \& BAUMGARTEN, J. 2007. Ferns, frogs, lizards, birds and bats in forest fragments and shade cacao plantations in two contrasting landscapes in the Atlantic forest, Brazil. Biodivers. Conserv. 16(8):2335-2357.

FRANÇA, F.G.R. \& ARAÚJO, A.F.B. 2006. The conservation status of snakes in central Brazil. South Am. J. Herpetol. 1(1):25-36.
MANTOVANI, W. 2000. Cobertura Vegetal do Município de São Paulo. Prefeitura do Município de São Paulo, São Paulo. Relatório Interno da Secretaria do Meio Ambiente da Prefeitura do Município de São Paulo. Atlas Ambiental do Município de São Paulo. 32 p.

MARQUES, O.A.V., MARTINS, M. \& ABE, A.S. 1998. Estudo diagnóstico da diversidade de répteis do Estado de São Paulo. In Biodiversidade do Estado de São Paulo: Síntese do conhecimento ao final do século XX (R.M.C. Castro, org). FAPESP, São Paulo, p. 29-38.

MARQUES, O.A.V. 1998. Composição Faunística, História Natural e Ecologia de Serpentes da Mata Atlântica, na Região da Estação Ecológica Juréia-Itatins, SP. Tese de Doutorado, Universidade de São Paulo, São Paulo.

MARQUES, O.A.V. \& SAZIMA, I. 2004. História natural dos répteis da Estação Ecológica Juréia-Itatins. In Estação Ecológica Juréia-Itatins: Ambiente Físico, Flora e Fauna (O.A.V. Marques \& W. Duleba, eds). Holos Editora, Ribeirão Preto, p. 257-277.

MARQUES, O.A.V., ETEROVIC, A. \& SAZIMA, I. 2004. Snakes of the Brazilian Atlantic Forest: An Illustrated Field Guide for the Serra do Mar range. Holos Editora, Ribeirão Preto.

MARQUES, O.A.V., SAWAYA, R.J., STENDER-OLIVEIRA, F. \& FRANÇA, F.G.R. 2006. Ecology of the colubrid snake Pseudablabes agassizii in south-eastern South America. Herpetol. J. 16:37-45.

MARTINS, M. 1991. The lizards of Balbina, central Amazonia, Brazil: qualitative analysis of resource utilization. Stud. Neotr. Fauna and Environm. 26(3):179-190.

MARTINS, M. 1994. História natural de uma taxocenose de serpentes de mata na região de Manaus, Amazônia Central, Brasil. Tese de Doutorado, Universidade Estadual de Campinas, Campinas.

MARTINS, M. \& OLIVEIRA, M.E. 1999. Natural History of Snakes in Forests of The Manaus Region, Central Amazonia, Brazil. Herpetol. Nat. Hist. 6(2):78-150.

NOGUEIRA, C.C. 2006. Diversidade e padrões de distribuição da fauna de lagartos do Cerrado. Tese de Doutorado, Universidade de São Paulo, São Paulo.

NOGUEIRA, C., VALDUJO, P.H. \& FRANCA, F.G.R. 2005. Habitat variation and lizard diversity in a Cerrado area of Central Brazil. Stud. Neotrop. Fauna Environ. 40(2):105-112.

NOGUEIRA, C., COLLI, G.R. \& MARTINS, M. 2009. Local richness and distribution of the lizard fauna in natural habitat mosaics of the Brazilian Cerrado. Austral Ecol. 34:83-96.

PAPAVERO, N. \& TEIXEIRA, D.M. 2007. A Fauna de São Paulo nos séculos XVI a XVIII nos textos dos cronistas, missionários e relatos maçoneiros. Editora EDUSP, São Paulo.

PARPINELLI, L. 2008. História Natural da cobra-cega Liotyphlops beui (Anomalepididae). Dissertação de Mestrado, Universidade Estadual Paulista, São José do Rio Preto.

PARPINELLI, L. \& MARQUES, O.A.V. 2008. Seasonal and daily activity in the Pale-headed Blindsnake Liotyphlops beui (Serpentes: Anomalepididae) in southeastern Brazil. South Amer. J. Herpetol. 3(3):207-212.

PETRONE, P. 1995. Aldeamentos paulistas. Editora da Universidade de São Paulo, São Paulo.

PREFEITURA DO MUNICÍPIO DE SÃO PAULO. 2002. Fase I: Diagnóstico e bases para a definição de políticas públicas para as áreas verdes no Município de São Paulo, Sec. Mun. do Meio Ambiente, Sec. Mun. de Planejamento Urbano São Paulo. In Atlas Ambiental do Município de São Paulo (H. Takiya, ed). São Paulo.

PUORTO, G., LAPORTA-FERREIRA, I.L. \& SAZIMA, I. 1991. Serpentes na selva de pedra. Ciência Hoje, 13(76):66-67.

RHODIN, A.G.J., MITTERMEIER, R.A. \& ROCHA-E-SILVA, R. 1982. Distribution and taxonomic status of Phrynops hogei, a rare chelid turtle from Southeastern Brazil. Copeia, 1982(1):179-181.

RIBAS, E.R. \& MONTEIRO-FILHO, E.L.A. 2002. Distribuição e habitat das tartarugas de água-doce (Testudines, Chelidae) do estado do Paraná, Brasil. Biociências, 10(2):15-32. 
RODRIGUES, M.T. 1990. Os lagartos da floresta Atlântica distribuição atual e pretérita e suas implicações para estudos futuros. In II Simpósio sobre ecossistemas da costa sul brasileira: Estrutura, manejo e função. Academia de Ciências do Estado de São Paulo, São Paulo, p. 404-410.

SAWAYA, R.J. 2004. História Natural e Ecologia das Serpentes de Cerrado da Região de Itirapina - SP. Tese de Doutorado, Universidade Estadual de Campinas, Campinas.

SAWAYA, R.J., MARQUES, O.A.V. \& MARTINS, M. 2008. Composition and natural history of a Cerrado snake assemblage at Itirapina, São Paulo state, southeastern Brazil. Biota Neotropica, 8(2):129-151.

SAZIMA, I. \& ABE, A.S. 1991. Habits of five Brazilian snakes with coralsnake pattern, including a summary of defensive tactics. Stud. Neotr. Fauna and Environm. 26(3):159-164.

SAZIMA, I. \& HADDAD, C.F.B. 1992. Répteis da Serra do Japi: notas sobre história natural. In História natural da Serra do Japi: Ecologia e preservação de uma área florestal no sudeste do Brasil. (P.C. Morellato, org). Ed. UNICAMP; FAPESP, Campinas, p. 212-236.

SAZIMA, I. \& MANZANI, P.R. 1995. As cobras que vivem numa reserva florestal urbana. In Ecologia e preservação de uma floresta tropical urbana: Reserva de Santa Genebra. (P.C. Morellato \& H.F. Leitão-Filho, eds). Ed. UNICAMP, Campinas, p. 78-82.

SILVA, L.O. 1993. Subsídios para Formulação de uma Política para Áreas Verdes no Município de São Paulo. In A Questão Ambiental Urbana: Cidade de São Paulo (J.R. Phillipi, ed). Secretaria Municipal do Verde e do Meio Ambiente, São Paulo, p. 425-449.
SILVANO, D.L., COLLI, G.R., DIXO, M.B.O., PIMENTA, B.V.S. \& WIEDERHECKER, H.C. 2005. Anfíbios e Répteis. In Fragmentação de Ecossistemas: Causas, Efeitos sobre a Biodiversidade e Recomendações de Políticas Públicas (D.M. Rambaldi \& D.A.S. Oliveira, orgs). MMA; SBF, Brasília, p. 183-200.

SOUZA, F.L. 2004. Uma revisão sobre padrões de atividade e alimentação de cágados brasileiros (Testudines, Chelidae). Phyllomedusa, 3(1):15-27.

STRÜSSMANN, C. \& SAZIMA, I. 1993. The assemblages of the Pantanal at Poconé western Brazil: faunal composition and ecology summary. Stud. Neotr. Fauna Environm. 28(33):157-168.

USTERI, A., 1911. Flora der Umgebung der State São Paulo in Brasilien. Verlag \& Gustav Ficher, Jena.

VANZOLINI, P.E. 1948. Notas sôbre os ofídios e lagartos de Emas, no Município de Pirassununga, estado de São Paulo. Rev. Bras. Biol. 8(3):377-400

VITT, L.J. \& VANGILDER, L.D. 1983. Ecology of snake community in the northeastern Brazil. Amphib- Reptil. 4(2-4):273-296.

ZIMMERMAN, B.L. \& RODRIGUES, M.T. 1990. Frogs, snakes and lizards of INPA-WWF reserves near Manaus, Brazil. In Four neotropical rain forest (A.H. Gentry, ed). Yale University Press, New Haven, p. 426-454.

Recebido em 12/09/08 Versão Reformulada recebida em 29/04/09 Publicado em 19/05/09 
Apêndice 1. Lista do material examinado nas coleções do Museu de Zoologia da Universidade de São Paulo (MZUSP) e do Instituto Butantan (IBSP). CRIBSP

Appendix 1. List of specimens examined in collections of Museu de Zoologia da Universidade de São Paulo (MZUSP) and Instituto Butantan (IBSP).

Quelonia: Acanthochelys spixii Ipiranga (MZUSP 302); Hydromedusa tectifera (MZUSP 330, 341, 490, 509, 774, 4066, 4201). Lacertilia: Ameiva ameiva (MZUSP 89524); Anisolepis grilii (MZUSP 286, 561, 4494, 4495, 4519), Butantã (MZUSP 4518), Caxingui (MZUSP 3616), Indianópolis (MZUSP 4550), Ipiranga (MZUSP 2796), Vila Galvão (MZUSP 4493); Colobodactylus taunayi (MZUSP 40083); Ecpleopus gaudichaudi (MZUSP 1966); Enyalius iheringiii (MZUSP 3140, 3141, 36665), Santo Amaro (MZUSP 4254); Enyalius perditus (MZUSP 835), Bororé (MZUSP 92269, 92270), Butantã (MZUSP 13594); Hemidactylus mabouia (MZUSP 2755, 19505, 46378); Heterodactylus imbricatus Parque Estadual da Cantareira-Núcleo Pedra Grande (MZUSP 89185, 89186); Mabuya dorsivittata (MZUSP 90002), Alto de Pinheiros (MZUSP 52446), Cidade Universitária (MZUSP 39474), Educandário (MZUSP 4687), Heliópolis (MZUSP 36550, 36551, 36552, 36553, 36554, 36555), Ipiranga (MZUSP 2863, 2864, 2865), Itaquera (MZUSP 56705), Jaguaré (MZUSP 6922), Lapa (MZUSP 42779), Represa do Guarapiranga (MZUSP 3992), Rio Pinheiros (MZUSP 40757); Mabuya frenata (MZUSP 389, 6928, 7329, 7333); Ophiodes fragilis Cantareira (MZUSP 3707), Cidade Universitária (MZUSP 36346, 55530), Guaianases (MCP 6887), Ipiranga (MZUSP 2842), Itaim (MZUSP 10317), Parelheiros (MZUSP 91597), Pirituba (MZUSP 57853), Rio Pequeno (MZUSP 42780), Santo Amaro (Campo Limpo) (MZUSP 44693), São Paulo (LAROF 342, 347), (MCP 6884, 6885, 6886, 7355, 7359), (MZUSP 475, 480, 4328, 4329, 23854, 78958); Ophiodes striatus (MZUSP 468, 473, 474, 486, 763, 8436, 79690); Pantodactylus quadrilineatus (MZUSP 2280, 40751, 40752, 40753, 40754), Cidade Universitária (MZUSP 54840, 55592), Sumaré (MZUSP 4787); Pantodactylus schreibersii Alto de Pinheiros (MZUSP 40732, 40733), Brás (MZUSP 40734), Heliópolis (MZUSP 36615, 36616, 36617, 36618, 36619, 36620, 36621, 36622, 36623, 36624, 36625), Ipiranga (MZUSP 47888); Polychrus acutirostris Butantã (MZUSP 4388, 4391); Tropidurus itambere (IBSPH $462-$ 468); Tupinambis merianae (MZUSP 2648, 4873, 29607, 29608), Interlagos (MZUSP 88855), Ipiranga (MZUSP 11968), Parque Estadual da Cantareira-Núcleo Pedra Grande (MZUSP 88182), Taipas (MZUSP 8482); Urostrophus vautieri (MZUSP 2549, 3190, 4460, 4461), Parque do Estado (MZUSP 92183), Represa do Guarapiranga (MZUSP 8404), Santo Amaro (MZUSP 76284, 78202, 78203, 78204, 78205, 78206). Amphisbaenia: Amphisbaena alba (MZUSP 77582, 91344); Amphisbaena dubia (MZUSP 87767, 87768, 87769, 87771, 89663), Santo Amaro (MZUSP 60424); Amphisbaena hogei (MZUSP 6633, 6634, 6675, 6691, 17003); Amphisbaena mertensi (MZUSP 8770, 79685), Alto de Pinheiros (MZUSP 90931), Vila Gustavo (MZUSP 88661); Amphisbaena trachura Pinheiros (IBSPH 444); Cercolophia roberti (MZUSP 755, 1257, 1937, 1946-47); Leposternon microcephalum (MZUSP 77011, 77517, 79688, 88894, 89140), Jabaquara (MZUSP 79348). Serpentes: Apostolepis assimilis Parque São Domingos (IBSP 59332, 59533, 66859), Pirituba (IBSP 60762, 67170, 67905, 68650), Santo Amaro (IBSP 1636, 24180, 53727); Apostolepis dimidiata Cerqueira Cesar (IBSP 12745), Jardim Brasil (IBSP 56014); Atractus pantostictus (IBSP 63496, 64100), Jaraguá (IBSP 42404, 55740, 59414, 59766, 60694, 60697); Atractus reticulatus (IBSP 62394, 62395, 62396, 64071, 64495), Brooklin (IBSP 20718, 28809, 28810), Vila Maria (IBSP 842); Atractus zebrinus Parelheiros - Núcleo Curucutu (IBSP 76785); Boiruna maculata (IBSP 9388, 23723), Barro Branco (IBSP 16845), Caxingui (IBSP 15666), Cidade Jardim (IBSP 25652), Jardim Bonfiglioli (IBSP
45926), Jardim Morumbi (IBSP 21536); Bothrops jararaca (IBSP 17741), Butantã (IBSP 2744), Cantareira (IBSP 69965), Horto Florestal (IBSP 25806), Parada de Taipas (IBSP 69981), Parelheiros (IBSP 69884, 69982, 69983), Represa do Guarapiranga (IBSP 69876), Santo Amaro (IBSP 12915); Chironius bicarinatus (IBSP 16138, 17044, 21524), Bairro do Pari (IBSP 60296), Butantã (IBSP 66761), Cidade Tiradentes (IBSP 62996), Interlagos (IBSP 55640), Pico do Jaraguá (IBSP 62162), Santo Amaro (IBSP 63899); Chironius exoletus (IBSP 5580, 16706, 24585, 53826), Parelheiros (IBSP 23156, 23157), Pico do Jaraguá (IBSP 63467); Chironius quadricarinatus Pinheiros (IBSP 822), São Miguel Paulista (IBSP 13818); Clelia plumbea (IBSP 27285, 29013, 30023, 30312); Clelia quimi Butantã (IBSP 380, 630, 711, 880, 1350, 9074, 11327), Vila Indiana (IBSP 33131); Crotalus durissus Bairro Teotônio Vilela (IBSP 69973), Jaraguá (IBSP 54614, 54664, 55155, 63860); Echinanthera amoena (IBSP 40233), Horto Florestal (IBSP 3093, 25632), Vila Matilde (IBSP 56479); Echinanthera cephalostriata Jd. João XXIII (IBSP 72641), (IBSP 2296, 28133, 56934); Echinanthera cyanopleura (IBSP 877, 56412); Echinanthera undulata (IBSP 5422, 6036, 7019, 9575, 57746, 58477, 60159), Água Funda (IBSP 60178), Bairro Jaceguava (IBSP 60556); Elapomorphus quinquelineatus (IBSP 49786), Horto Florestal (IBSP 46346, 55135), Jaçanã (IBSP 69260); Erythrolamprus aesculapii Bairro Campo Grande (IBSP 7432), Horto Florestal (IBSP 8750, 28045), Jaraguá (IBSP 61998), Pirituba (IBSP 19034), Vila Galvão (IBSP 6076); Gomesophis brasiliensis (IBSP 9648, 10420), Butantã (IBSP 3083, 5200), Caxingui (IBSP 22105), Santo Amaro (IBSP 19694); Helicops carinicaudus (IBSP 57659, 66997); Helicops modestus Freguesia do Ó (IBSP 28510), Guarapiranga (IBSP 62145), Ipiranga (IBSP 11867, 22609), Santo Amaro (IBSP 32520), São Miguel Paulista (IBSP 27499), Vila dos Remédios (IBSP 56255); Liophis almadensis (IBSP 6424, 10036, 10520, 10582, 62322), Butantã (IBSP 42178), Morumbi (IBSP 27136), Pinheiros (IBSP 5516, 10249), Santo Amaro (IBSP 16091), Vila Galvão (IBSP 4780); Liophis atraventer Perdizes (IBSP 25295); Liophis flavifrenatus Bairro Cidade Jardim (IBSP 5962), Butantã (IBSP 28103), Itaquera (IBSP 10206, 10207, 10208, 10209), São Miguel Paulista (IBSP 26525, 26526, 26527, 26528, 26678, 28104); Liophis miliaris Itaim-Bibi (IBSP 18169, 18170, 18171), Itaquera (IBSP 23362), Santo Amaro (IBSP 16092, 17203, 29424), São Miguel Paulista (IBSP 11863), Vila dos Remédios (IBSP 876), Vila Nova Conceição (IBSP 58309); Liophis jaegeri Itaquera (IBSP 10198, 10199), Morumbi (IBSP 18920), Santana (IBSP 191), Santo Amaro (IBSP 194, 1322, 8042, 27631, 46653), Tremembé (IBSP 10368); Liophis poecilogyrus (IBSP 5603, 27132), Santo Amaro (IBSP 4328, 22785, 32140, 56640), São Miguel Paulista (IBSP 8224); Liophis reginae Butantã (IBSP 27639, 33242), Santo Amaro (IBSP 24901), Vila Indiana (IBSP 28112); Liophis typhlus Bairro Guarapiranga (IBSP 58342, 62285), Itaquera (IBSP 30758), Jardim Arpoador (IBSP 61961), Perus (IBSP 6657), Santana (IBSP 28458), Santo Amaro (IBSP 16223, 18854, 60530); Liotyphlops beui Perdizes (IBSP 62944), Pirituba (IBSP 62942, 63638, 64621, 68356); Mastigodryas bifossatus Bairro Cidade Jardim (IBSP 56077), Parelheiros (IBSP 31272), Santo Amaro (IBSP 16088); Micrurus corallinus (IBSP 6970, 61476), Brooklin (IBSP 42256), Butantã (IBSP 33020), Cidade Universitária (IBSP 61425), IBSPirapuera (IBSP 37464), Jaguaré (IBSP 7558), Parelheiros (IBSP 50236), Pirituba (IBSP 41712), Santo Amaro (IBSP 34484, 45479); Micrurus decoratus (IBSP 6884), Bairro Ponte Lavrada (IBSP 7717), Butantã (IBSP 1816-fornecedor- 
Vital Brazil filho); Micrurus frontalis (IBSP 8822), Serra da Cantareira (IBSP 44337), Vila Ipojuca (IBSP 42390); Micrurus lemniscatus Butantã (IBSP 5824), Jardim Bonfiglioli (IBSP 30947), Santo Amaro (IBSP 9933); Oxyrhopus clathratus (IBSP 50064, 60573, 62499), Horto Florestal (IBSP 52415), Pinheiros (IBSP 18600), Santo Amaro (IBSP 54457), Vila Albertina (IBSP 57324); Oxyrhopus guibei (IBSP 387, 2760, 5519, 16089, 16189, 20991, 21937, 44612, 52530, 57586, 59623, 60543); Phalotris mertensi (IBSP 57340); Philodryas aestivus (IBSP 736, 3171, 6778, 7536, 7914, 17948, 23350, 28560, 29342, 54943); Philodryas mattogrossensis Pico do Jaraguá (IBSP 58308); Philodryas olfersii (IBSP 2756, 29182, 42179, 52531, 53752, 64013), Pirituba (IBSP 67501); Philodryas patagoniensis (IBSP 2680, 10588, 13155, 18165, 28312, 42180, 53557, 58729, 64221, 67275); Phimophis guerini (IBSP 43463); Pseudoboa serrana $\mathrm{B}^{\circ}$ Campo Grande(IBSP 5874); Sibynomorphus mikanii (IBSP 3277, 17942, 41221, 41262, 51863, 52525, 62119), Butantã (IBSP 68384), Morumbi (IBSP 43292); Sibynomorphus neuwiedi (IBSP 13009, 16350, 43313, 45809); Simophis rhinostoma (IBSP 168, 2935, 2936, 2940, 3320, 52515, 52523), Guarapiranga (IBSP 45670), Lapa (IBSP 42327); Siphlophis longicaudatus Santo Amaro (IBSP 54957); Siphlophis pulcher Tucuruvi (IBSP 49849); Sordellina punctata Butantã (IBSP 6791), Jardim Aeroporto (IBSP 49190), Parelheiros (IBSP 34286, 34290, 42914), Santo Amaro (IBSP 22936, 22937, 33201); Spilotes pullatus (IBSP 2769), Butantã (IBSP 5122, 5123), Guarapiranga (IBSP 19591), Santana (IBSP 6083); Taeniophallus affinis Água Funda (IBSP 69610, 70245, 70246), Vila Nova Conceição (IBSP 48605); Taeniophallus bilineatus (IBSP 190), Butantã (IBSP 3250), Cidade Tiradentes (IBSP 68707), Santo Amaro (IBSP 41190, 53742, 54511); Taeniophallus occipitalis Brooklin (IBSP 17837), Itaquera (IBSP
29509, 53744), Jaguaré (IBSP 19739), Parada de Taipas (IBSP 12873), Piqueri (IBSP 4765), Vila dos Remédios (IBSP 10842); Taeniophallus persimilis Cantareira (IBSP 71893); Tantilla melanocephala Bairro Catanduva (IBSP 43642), Jardim Martinica (IBSP 55623, 55624), Pinheiros (IBSP 2921, 3325, 27813), Riviera Paulista (IBSP 55022), Santo Amaro (IBSP 122, 734, 21687), Vila Carrão (IBSP 10272, 10273); Thamnodynastes cf. nattereri (IBSP 896), Parque da Água Funda (IBSP 24540, 24541); Thamnodynastes hypoconia Itaim (IBSP 18182, 18183), Pirituba (IBSP 11189), Santo Amaro (IBSP 12304, 16090, 30384), São Miguel Paulista (IBSP 26529, 26530), Tremembé (IBSP 10369); Thamnodynastes strigatus Jardim Peri-Peri (IBSP 28820), Parelheiros (IBSP 59949), Pirituba (IBSP 10962, 11411), Santo Amaro (IBSP 21938); Tomodon dorsatus Interlagos (IBSP 62050), Parelheiros (IBSP 52467, 59357), Pico do Jaraguá (IBSP 31709), Santo Amaro (IBSP 59398, 59606, 61348, 68035); Tropidodryas serra (IBSP 370, 25348);Bela Vista (IBSP 24449), Butantã (IBSP 3271); Tropidodryas striaticeps (IBSP 9912, 9921, 59983, 61796), Água Fria (IBSP 57497), Água Funda (IBSP 53672, 54939, 56454), Guaianases (IBSP 23284), Jabaquara (IBSP 60156), Parque Nova FrIBSPurgo (IBSP 43198), Perus (IBSP 44660), Pico do Jaraguá (IBSP 57705); Tropidophis paucisquamis Vila Jaguara (IBSP 74110); Uromacerina ricardinii Bom Retiro (IBSP 32046), Butantã (IBSP 393, 394, 398, 710, 2920, 3266), Horto Florestal (IBSP 24053), Parelheiros (IBSP 26868), Vila Nova Conceição (IBSP 24455); Waglerophis merremii (IBSP 52497, 52500, 52511), São Mateus (IBSP 53547), Vila Progresso (IBSP 53631); Xenodon neuwiedii Água Funda (IBSP 24959, 25969), Jardim Shangrilá (IBSP 59421), Pirituba (IBSP 59989), Santo Amaro (IBSP 59988, 60934, 60935), Serra da Cantareira (IBSP 25950, 25951, 25964, 25965), Vila Jaguara (IBSP 61981). 